\title{
The scope of nanoparticle therapies for future metastatic melanoma treatment
}

\author{
Francesca Baldelli Bombelli, Carl A Webster, Marc Moncrieff, Victoria Sherwood
}

Metastatic melanoma is a highly aggressive malignancy that has traditionally been very diffi cult to treat. However after decades of basic research into the signal transduction pathways that promote cancer cell survival, chemoresistance, growth, and crosstalk with the immune system, targeted therapies have now been developed that offer improved survival for patients with metastatic melanoma. Some of the most promising therapies that have been developed include ipilimumab, an anti-cytotoxic $\mathrm{T}$ lymphocyte antigen 4 antibody that enhances T-cell activity in the tumour and selective BRAF inhibitors, such as vemurafenib that blocks tumour cell proliferation in patients with activating BRAF mutations. Although these treatments off er substantial hope for patients, they are not without their drawbacks which include adverse side-eff ects, drug resistance, and eventual relapse. Nanotherapeutics holds significant promise to circumvent these shortcomings and has the additional advantage of potentially functioning as a diagnostic device. We will discuss the scope of the use of such multimodal nanoparticles for melanoma treatment and ask whether such particles can off er patients with metastatic melanoma improved prognoses for the future.

\section{Introduction}

The incidence of melanoma is rising faster than that of any other cancer and has been increasing steadily for the past four decades ${ }^{1,2}$ to the extent that it is now reaching epidemic proportions in white populations worldwide. Melanoma is a malignancy derived from melanocytes (pigment-producing cells) and is now the sixth most frequently diagnosed cancer in human beings. Unlike many other cancers, melanoma tends to affect young people-55\% of patients are younger than 65 years at diagnosis. Once melanoma has developed into late-stage metastatic disease, it is notoriously difficult to treat, resulting in high mortality. Hence, the 3-year overall survival of patients with unresectable melanoma is poor (lower than 15\% with conventional treatments). ${ }^{3}$

Conventional treatments for melanoma include surgery, chemotherapy, and immunotherapy; of these, surgery provides optimum results for patients at all stages of the disease. Unfortunately for those patients presenting with metastases, disseminated tumours in distant organs are often unresectable and until now very few therapeutic options have been available to these patients. However, since the molecular mechanisms of melanoma cell survival have been unravelled, several promising approaches for the treatment of metastatic disease have emerged. New drugs have been developed that have achieved prominent clinical responses in phase 3 trials. ${ }^{46}$ Some of the most promising therapies include ipilimumab, an anti-cytotoxic $\mathrm{T}$ lymphocyte antigen 4 antibody that enhances T-cell activity in the tumour, and selective BRAF inhibitors (fi gure 1). The ongoing development of new drugs for metastatic melanoma is so momentous that two new therapies (the BRAF inhibitor dabrafenib and the MEK inhibitor trametinib) have recently been approved by the US Food and Drug Administration (FDA), to become the third and fourth new treatments to be approved for late-stage melanoma in the past 24 months. Although these targeted therapies have begun to show some clinical promise for patients with melanoma, they are limited by their low response and high relapse rates. Future efforts will need to address these issues and should focus in particular on the development of effective combination therapies aimed at simultaneously targeting tumourderived coexistent mechanisms and circumventing resistance strategies.

We believe that one especially advantageous approach is the development of melanoma-specific nanotherapeutics. Nanoparticles are defined as nano-scaled systems of diverse shapes made of inorganic materials, organic materials, or both, ranging from $1 \mathrm{~nm}$ to $200 \mathrm{~nm}$ in size. Theranostic (a portmanteau of therapeutic and diagnostic) nanoparticles function simultaneously as therapeutic and detection devices, offering multimodal platforms that could greatly improve patient prognosis in a range of diseases.? Indeed, nanomedicine holds the promise of revolutionising the treatment of patients with cancer in the future. Several nanoparticles are being engineered

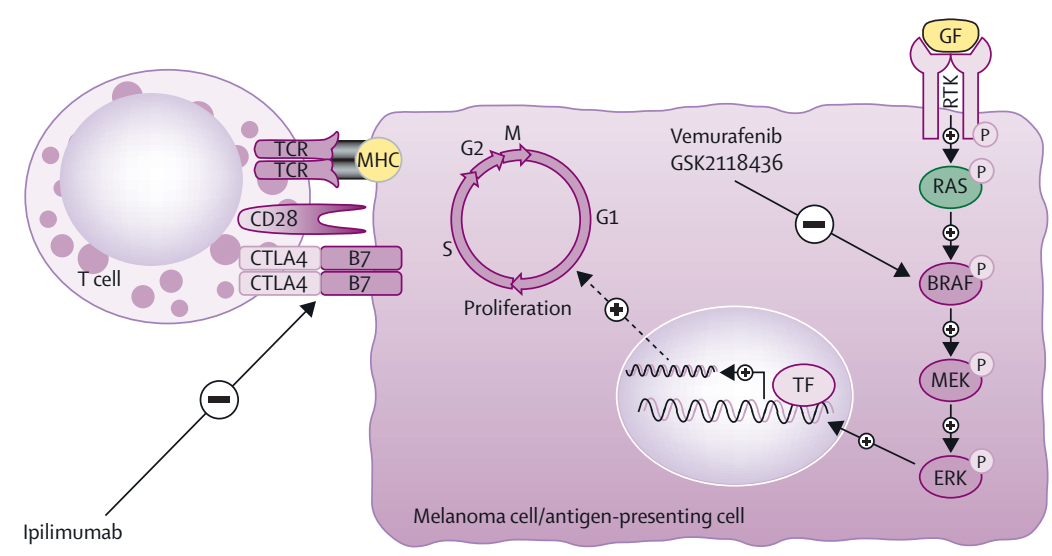

Figure 1: Present strategies to manage advanced stage melanomas include inhibition of the mutant form of BRAF kinase (a common mutation being BRAF ${ }^{\mathrm{V} 600 \mathrm{E}}$; vemurafenib and GSK2118436) and T-cell activation (ipilimumab) $\mathrm{TCR}=\mathrm{T}$-cell receptor. $\mathrm{GF}=$ growth factor. $\mathrm{MHC}=$ major histocompatibility complex. $\mathrm{RTK}=$ receptor tyrosine kinase. CTLA4=cytotoxic T lymphocyte antigen $4 . \mathrm{TF}=$ transcription factor.

\author{
School of Pharmacy, University \\ of East Anglia, Norwich, \\ Norfolk, UK \\ ( $\mathrm{F}$ Baldelli Bombelli PhD, \\ CA Webster BSc, \\ V Sherwood PhD); CEN- \\ European Centre For \\ Nanomedicine, $\mathrm{C} / \mathrm{O}$ \\ Dipartimento di Chimica \\ Materiali ed Ingegneria \\ Chimica "Giulio Natta", \\ Politecnico di Milano, Milan, \\ Italy (F Baldelli Bombelli); and \\ Norfolk and Norwich \\ University Hospital, Norwich, \\ Norfolk, UK \\ (M MoncrieffFRCS [Plast]) \\ Correspondence to: \\ Dr Victoria Sherwood, School of \\ Pharmacy, University of East \\ Anglia, Norwich Research Park, \\ Norwich, Norfolk NR4 7JT, UK \\ v.sherwood@uea.ac.uk
}


and tested for tumour imaging and targeted drug delivery for melanoma. The aim of this Review is to emphasise the potential benefi ts of nanoparticles for diagnosing and treating melanoma, and to outline existing approaches in developing nanotechnologies for this purpose. Ultimately, we will propose a rationale for the design considerations needed for the development of highly eff ective nanotherapeutic systems for melanoma treatment and their clinical use.

\section{Nanotechnology-based therapeutic approaches to} melanoma treatment

\section{Drug delivery nano-based systems for melanoma}

Medical research has been implementing strategies for improved melanoma treatment since the 1960s, but many substantial steps in improving overall survival in patients have only been made in recent years (figure 2). Furthermore, huge advances have been achieved in the identification of several key molecular targets for melanoma treatment, to add to the already exploited anti-CTLA4 and anti-BRAF strategies. Several comprehensive reviews of this topic are available, ${ }^{8-10}$ but we have summarised some of the most promising targets for the development of future treatments for melanoma (appendix p 1). However, as with the limitations of vemurafenib and ipilimumab, the individual targeting of any one of these deregulated molecules would probably lead to the selection of resistant tumour cell populations and eventual patient relapse. Thus, the use of several drugs in combination is one of the main strategies to prevent the development of multidrug resistance in tumours.

Multidrug resistance is caused by complex molecular mechanisms that are difficult to predict in patients. Two frequently identified drug-inhibiting mechanisms are the overexpression of multidrug transporters (upregulation of drug efflux) and the simultaneous accumulation of many prosurvival mutations. ${ }^{11}$ Combination therapies of conventional chemotherapeutics can provide encouraging results in vitro that are not always reproduced in clinical trials. ${ }^{12}$ The main reason for this incongruence is related to the different pharmacokinetic properties of the combinatorial drugs, which can have specific metabolic pathways with diverse biochemical fates in patients. This situation makes it very challenging to design the correct formulation, dosing, and administration route for such therapies. Although multitargeted strategies are necessary to overcome multidrug resistance mechanisms and several studies have investigated effective combinatorial approaches for melanoma treatment, ${ }^{13-16}$ the clinical potential of such formulations is still unknown and could prove to be disappointing for the reasons discussed previously.

Nanoparticles can act as multimodal platforms, which accommodate various clinically relevant properties for cancer treatment. In fact, engineered nanomaterials can be designed to function as contrast agents for existing and emerging imaging techniques, and to simultaneously serve as delivery devices for anticancer drugs. ${ }^{17}$ Nanoparticulate systems for anticancer treatment have conventionally been classified into either organic or inorganic nanomaterials (figure 3), but many hybrid nanomaterials have been developed to obtain further advanced materials that can work as theranostic platforms. ${ }^{18,19}$ In view of the ever-increasing use of diverse nanoscaled therapeutics (figure 3 ) and the identification of a large array of potential therapeutic target molecules

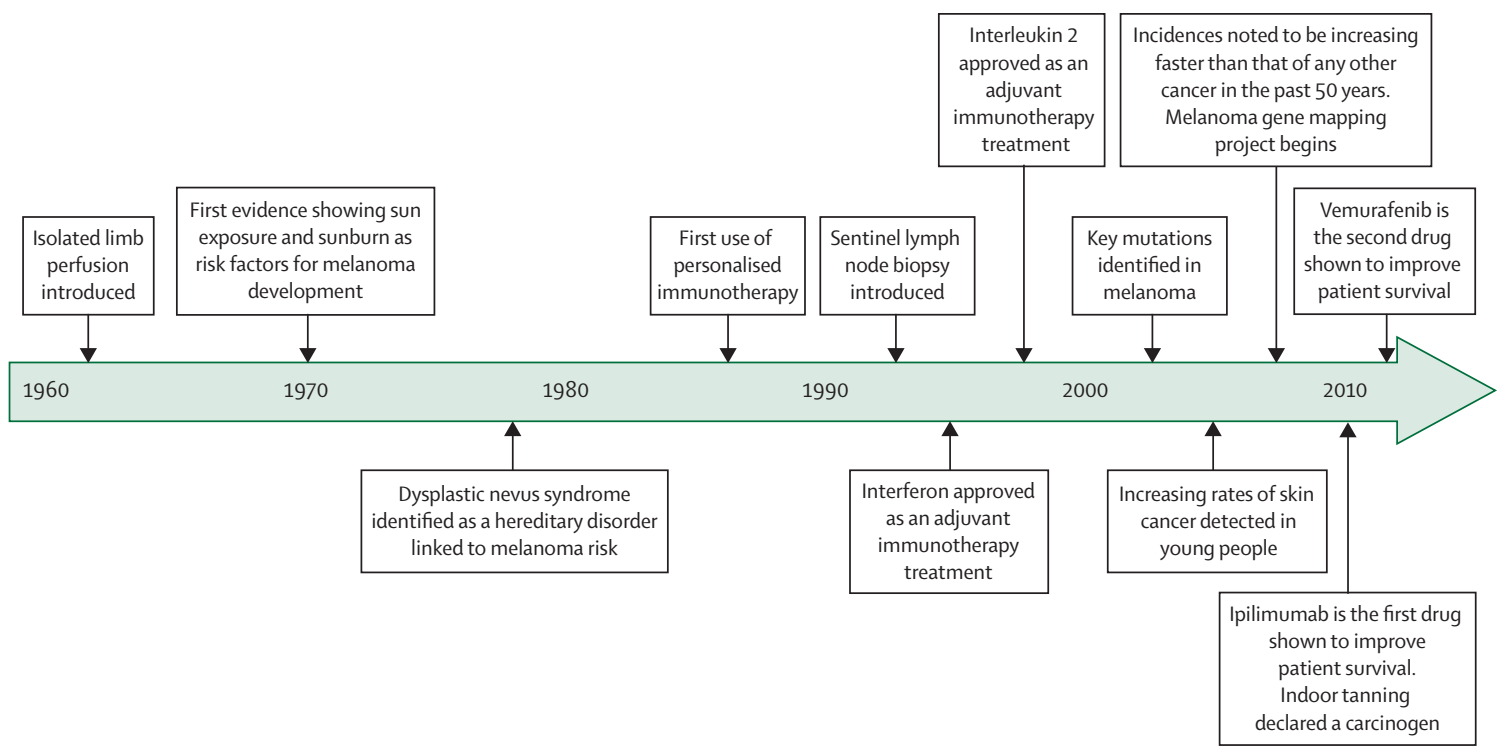


for melanoma (appendix p 1), now is clearly an ideal time for the development of nanomedicines that incorporate multifunctional benefits to treat patients with melanoma.

Generally, the encapsulation of drugs in nanoparticles has been reported to improve the pharmacokinetic properties of the compounds. This finding is attributable to better tumour accumulation by passive and active targeting, sustained drug release, longer blood circulation times, and the fact that nanoparticles are taken up by cells through endocytosis, which carries drugs away from the cell membrane where there is a reduced chance of drug removal by pump efflux processes. ${ }^{20-22} \mathrm{~A}$ range of nanoparticle formulations could be envisaged to act as carriers of small-molecule drugs for melanoma treatment, including those that are used in conventional chemotherapeutic strategies for metastasis, such as dacarbazine. ${ }^{23}$

Another excellent example of the potential improvements achieved through nanotechnology is for taxanebased drug administration. Taxanes are effective chemotherapeutic drugs, but exhibit high toxicity associated with their pharmacological formulations and non-specific distribution, leading to severe side-effects in patients. ${ }^{24}$ Nanotechnologies have been designed that eliminate both of these issues by encapsulating taxane drugs in polymeric micelles, a commercially available formulation being a nanoparticle conjugate, in which paclitaxel is non-covalently complexed with human serum albumin. This product has been licensed for use by the US FDA in patients with breast cancer since $2005 .^{25}$ Several clinical trials have investigated the potential of albumin-bound paclitaxel for melanoma patients with unresectable tumours, either used individually (clinical trial for intraocular melanoma NCT00738361; table) or in combination with other drugs ${ }^{26}$ (clinical trials NCT00626405, NCT00404235, and NCT00462423), where the effects seem to be promising for standard chemotherapy-naive patients. A further taxane-based nanoformulation with a conjugate of docetaxel and a modified carboxymethylcellulose polymer ${ }^{27}$ was recently shown to have less toxic side-effects and improved efficacy on tumour burden compared with albumin-bound paclitaxel in a preclinical rodent model of melanoma, ${ }^{28}$ but whether these improvements can be translated to patients with melanoma remains to be studied.

Nanoparticles can also provide a delivery platform for a range of cytotoxic drugs, without the drawbacks of systemic toxicity caused by conventional chemotherapies. This idea has been shown for the fullerene-based delivery of the chemotherapeutic agent doxorubicin in animal models of melanoma, where the nanoparticle-drug conjugates had similar efficacy to free doxorubicin treatment, but with negligible toxic side-effects. ${ }^{29}$ Such toxic bombs can show improved anticancer effects compared with free drug, in addition to reduced toxicity, which is often attributed to sustained drug release in the tumour mass, ${ }^{28}$ as has previously been shown for docetaxel-loaded nanoparticles in murine models of melanoma. ${ }^{30}$

Many compounds could be loaded into nanoparticles, which is a fantastic opportunity to successfully deliver several anticancer drugs simultaneously. Nanoparticles can also be engineered to target the tumour site, releasing their cargos at specific locations with a reduction in the severe side-effects associated with conventional chemotherapies. In fact, a higher flexibility in the loading ratio of the drugs and better control of the sequential release allows for a synergic therapeutic action that can attack several aspects of tumour cell survival, thereby addressing the issue of multidrug resistance. ${ }^{31,32}$ One of the main reasons for the failure of many combinatorial therapies in clinical trials is the different metabolic
Organic nanoparticles

A

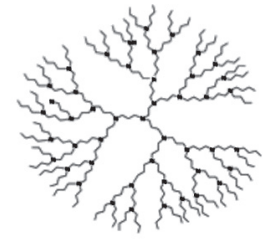

C

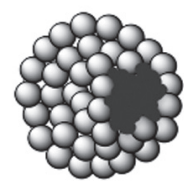

B

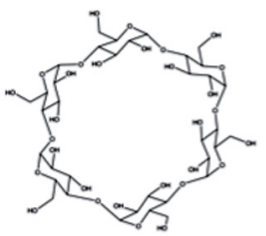

D

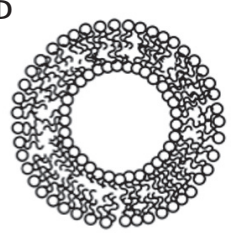

Inorganic nanoparticles

$\mathrm{E}$

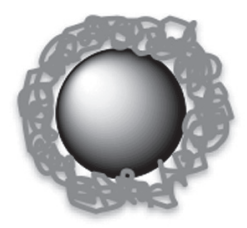

G

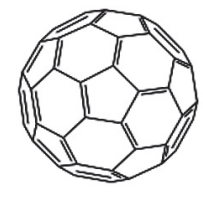

$\mathrm{F}$

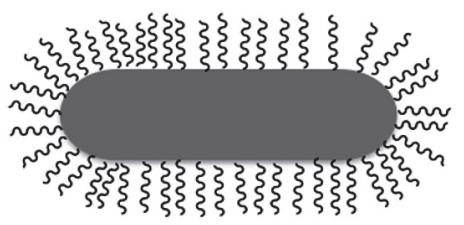

H

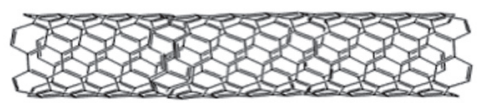

Figure 3: The most frequently used nanomaterials in oncology

Organic nanoparticles (left panel): (A) dendrimer, (B) cyclodextrin, (C) micelles, (D) liposomes. Inorganic nanoparticles (right panel): (E) core-shell nanoparticle, (F) nanorod, (G) fullerene, (H) carbon nanotube. 


\begin{tabular}{|c|c|c|c|c|}
\hline & Phase & Trial number & Disease & Outcome \\
\hline Albumin-stabilised paclitaxel nanoparticles & 2 & NCT00081042 & $\begin{array}{l}\text { Unresectable stage III and IV } \\
\text { melanomas }\end{array}$ & $\begin{array}{l}\text { Improved progression-free survival } \\
\text { compared with dacarbazine treatment alone }\end{array}$ \\
\hline Albumin-stabilised paclitaxel nanoparticles & 2 & NCT00738361 & $\begin{array}{l}\text { Unresectable metastatic } \\
\text { ocular melanoma }\end{array}$ & Unreported so far \\
\hline $\begin{array}{l}\text { Albumin-stabilised paclitaxel nanoparticles in } \\
\text { combination with carboplatin }\end{array}$ & 2 & NCT00404235 & $\begin{array}{l}\text { Unresectable stage IV } \\
\text { melanoma }\end{array}$ & $\begin{array}{l}\text { Improved overall survival compared with } \\
\text { ipilimumab }\end{array}$ \\
\hline $\begin{array}{l}\text { Albumin-stabilised paclitaxel nanoparticles in } \\
\text { combination with VEGF inhibitors }\end{array}$ & 2 & NCT00462423 & $\begin{array}{l}\text { Unresectable metastatic } \\
\text { melanoma }\end{array}$ & Unreported so far \\
\hline $\begin{array}{l}\text { Albumin-stabilised paclitaxel nanoparticles in } \\
\text { combination with VEGF inhibitors and carboplatin }\end{array}$ & 2 & NCT00626405 & $\begin{array}{l}\text { Unresectable stage IV } \\
\text { melanoma }\end{array}$ & $\begin{array}{l}\text { Improved overall survival compared with } \\
\text { patients treated with VEGF inhibitors and } \\
\text { temozolomide }\end{array}$ \\
\hline $\begin{array}{l}\text { Anti-RRM2 siRNA-loaded cyclodextrin polymer- } \\
\text { based nanoparticles, targeted to transferrin- } \\
\text { overexpressing cells }\end{array}$ & 1 & NCT00689065 & $\begin{array}{l}\text { Solid metastatic malignancies } \\
\text { including melanoma }\end{array}$ & $\begin{array}{l}\text { Successful reduction in RRM2 expression in } \\
\text { tumour tissue from treated patients }\end{array}$ \\
\hline $\begin{array}{l}\text { Docetaxel-loaded nanoparticles (BIND-014; study } \\
\text { to establish maximum tolerated dose) }\end{array}$ & 1 & NCT01300533 & $\begin{array}{l}\text { Solid metastatic malignancies } \\
\text { including melanoma }\end{array}$ & Recruiting \\
\hline
\end{tabular}

Table: Clinical trials for nanoparticle-based therapies in malignant melanoma

pathways of the drugs; thus, encapsulation in a nanocarrier circumvents this obstacle by protecting the drugs from being metabolised until they reach their target. Different nanoformulations have been used to simultaneously deliver combinations of diverse drugs such as anti-angiogenesis agents and chemotherapeutics, ${ }^{33}$ generally with better therapeutic effects than conventional combined therapies for the treatment of multidrugresistant tumours. Since multidrug resistance is a common problem in the effective treatment of melanoma, nanoparticles loaded with several drugs might offer a promising alternative for the treatment of this disease.

\section{Theranostic nanoparticles for melanoma}

Additional sophistication of the drug delivery system can also be engineered into nanoparticles to improve drug payload further, such as administration of pro-drugs with activating enzymes and drug release itself being triggered either by the tumour microenvironment or through applied stimuli such as electromagnetic fields, ultrasound, light, or radiofrequencies..$^{34}$ These techniques are especially useful to enhance nanoparticle-based drug delivery, since inefficient escape from endosomes and potential lysosomal inactivation can be problematic in nano-mediated drug delivery. Ultrasound exposure has been shown to stimulate the intracellular delivery of drugs by nanoparticles in melanoma cells, ${ }^{35-38}$ with clear implications for the pharmacodynamics of nanoparticle drug delivery in a clinical setting.

Exploitation of the low $\mathrm{pH}$ tumour microenvironment caused by enhanced aerobic glycolysis (a well characterised metabolic alteration of cancer cells) is a commonly used strategy for nanoparticle drug release and this approach has recently been investigated as a possibility for gene delivery to melanoma cells. ${ }^{39}$ Therapeutic nanoparticles have also been suggested to be able to stimulate changes to the melanoma tumour microenvironment itself, including enhancement of immunotherapy through targeted transfection of splenic dendritic cells for enhanced recognition of melanoma cells, ${ }^{40}$ or even angiogenic remodelling of the tumour neovasculature. ${ }^{4}$ Nanoparticle-based therapeutics are not limited to the delivery of small molecules, which are normally necessary for systemic chemotherapeutic treatments, but can be used to deliver large bioactive molecules such as proteins and nucleic acids, including small interfering RNAs (siRNAs). Nanoparticle-based siRNA delivery has been used to successfully downregulate tumorigenic targets in melanoma cells such as c-Myc ${ }^{42}$ and ribonucleotide reductase. $^{43}$ A cyclodextrin polymer-based nanoparticle called CALAA- $01^{44}$ was used in the first ever patient trial to systemically deliver siRNA molecules with a targeted delivery system to solid tumours (clinical trial NCT00689065; see table).

Hyperthermia therapy for cancer exploits exogenous heat to kill tumour cells, which tend to be more sensitive than healthy cells to increases in temperature. ${ }^{45}$ Thermoablation provides a drug-free strategy to destroy cancer cells, thereby providing enhanced treatment options for patients with melanoma and multidrug resistance, whose tumours could have acquired mechanisms to resist the effects of a range of drug types with diverse structural properties. Cell-specific multimodal nanotherapeutics offering thermal and chemotherapy or immune therapy simultaneously represent very promising drug delivery platforms to provide such a combined treatment. One promising strategy has been developed by $\mathrm{Lu}$ and colleagues ${ }^{46}$ in which in-vivo photothermal ablation obtained by means of infrared-irradiated targeted hollow gold nanospheres induced selective destruction of B16 melanoma cells. Porphyrin-coated magnetic iron or iron (II, III) oxide nanoparticles given both intratumorally and intra- 
venously in B16 tumour-bearing mice induced a substantial decrease in the tumour volume by thermoablation under exposure to an alternating magnetic field. ${ }^{47}$ Indeed, this approach provides a local hyperthermic eff ect in the tumour, resulting in apoptosis and damage to the melanoma cells and thereby triggering tumour regression. ${ }^{48}$ Recently, Jimbow and colleagues developed magnetically triggered, thermoactive nanoparticles functionalised with $\mathrm{N}$-propionyl and $\mathrm{N}$-acetyl derivatives of 4S-cysteaminylphenol, which are known to be specifi cally toxic to melanoma cells. They showed that the thermal eff ect not only strongly enhanced the reduction in tumour mass, but also induced an immune response to the melanoma cells. ${ }^{49}$

The same idea has been applied to develop targeted nanoparticles working as imaging devices for the detection of early-stage tumours. Melanoma-targeted gold nanocages have been used successfully in vivo as selective contrast agents for photoacoustic tomography, showing high specificity and greatly improved imaging resolution. $^{50}$ Additionally, integrin-binding (RGD peptide)functionalised radiolabelled silica dots, called c-dots, have been shown to accumulate selectively in melanoma tumours in mice. PET is an essential method for in-depth imaging analysis of metastatic tumours; the additional optical features provided by the fl uorescent dye embedded in the silica matrix of the nanoparticles enhanced the detection sensitivity to image lymphatic drainage patterns, particle clearance rates, and nodal metastases with use of a deep-red near-infrared fl uorescence imaging technique in a mini-swine model of melanoma. ${ }^{51}$

The detection of micrometastases with sentinel lymph node biopsy greatly improves regional control of the disease and substantially reduces long-term morbidity. ${ }^{52}$ Furthermore, sentinel lymph node biopsy imparts valuable and highly accurate prognostic information to the patient and treating clinician. ${ }^{3}$ On average, only $16 \%$ of patients have metastases in the regional nodes, and sentinel lymph node biopsy is an invasive procedure. Accordingly, $84 \%$ of patients are potentially exposed to the morbidity of a surgical procedure without deriving any therapeutic benefi $t$. A noninvasive assessment of the regional lymph nodes for micrometastases is limited by the spatial resolution of existing imaging techniques, and targeted nanoparticles could off er an elegant solution. For example, thermally cross-linked superparamagnetic iron oxide nanoparticles that do not bear surface-targeting ligands, but do have a thermal crosslinked Si-OH-containing polymer coating, can enhance MRI of melanoma lymph node metastasis in experimental models. ${ }^{53}$ RGD-coupled c-dots have also been suggested as suitable probes to measure metastatic spread of melanoma cells to sentinel lymph nodes, and design considerations for how nanoparticles can be functionalised to do this eff ectively have recently been reviewed.54 The successful use of nanoparticles as contrast imaging agents has been emphasised by studies in patients with breast cancer. In an ongoing clinical trial in the UK (ISRCTN35827879), which started at the beginning of 2012 (and is now at the end of phase 2), coated magnetic nanoparticles were injected subcutaneously into breast tissue to detect sentinel lymph nodes in patients with breast cancer. In view of the encouraging results of this trial, in April, 2013, an analogue study of melanoma patients scheduled for sentinel lymph node biopsy was started in the UK.

\section{Successful targeting of nanoparticles in patients with melanoma}

In melanoma, metastases occur locoregionally, initially to the lymph nodes and sometimes the intervening skin. Systemic spread to either one or several organs, such as the brain and visceral organs, can occur frequently and is usually fatal. The next generation of nanoparticles will need to address the challenge of targeting unresectable, metastasised, late-stage tumours, which will need the successful accomplishment of both tissue targeting to tumours in specifi c organs (largely passive targeting) and subsequent targeting to the cancer cells directly (active targeting), and clearly these considerations are specific to individual cancer types. Distinct strategies are needed to achieve both of these targeting requirements.

\section{Melanoma cell targeting}

Effective targeting of nanoparticles to specific cell types such as disseminated tumour cells poses a set of challenging obstacles for medical researchers. To achieve this aim at the cellular level, upregulated cell surface markers on tumour cells can be exploited for targeting by functionalisation of the nanoparticles with cognate targeting moieties that help interaction with tumourspecific markers. This idea is not new, since the use of targeted therapies in oncology has been increasing steadily during the past few decades, a highly effective example being trastuzumab antagonism of HER2 in very aggressive breast cancers. Several overexpressed cell surface markers have been discovered in melanoma cells (appendix p 2), which have promising therapeutic potential as nanoparticle targets. In-depth pharmacological investigations are needed to achieve optimum and specific binding affi nity between the targeting moiety and marker, without unwanted immunological effects. Moreover, none of these markers are purely melanoma selective-in fact, they are also expressed either by melanocytes or endothelial cells and therefore have possible associated undesired nanoparticle uptake by healthy cells. Thus, some toxicity will be associated with targeted melanoma nanoparticles, although this toxicity is anticipated to be less severe than that associated with conventional chemotherapies for the treatment of late-stage disease.

One especially promising approach for high-specificity nanoparticle targeting to melanoma cells has been to take advantage of the melanin pigmentation signalling 
pathway and use analogues of the $\alpha$-melanocyte stimulating hormone to target the melancortin-1 receptor (MC1R). MC1R is expressed on melanocytes and at an even higher level in melanoma cells, allowing for enhanced specificity of nanoparticle targeting to melanoma tumours in animals. ${ }^{46,50,55}$ The development of powerful, unbiased molecular approaches to map the proteome on cancer cell surfaces will undoubtedly reveal more novel targeting moieties on melanoma cells (such techniques include mass spectrometry, ribosome display, and phage display). However, as is the case with any tumour-targeted therapy, patients with melanoma would probably need biopsy testing for positive expression of specific nanoparticle-targeting cell surface markers before treatment, as is the case for other targeted therapies such as vemurafenib (for which BRAF mutation status must be established).

\section{Tissue targeting for melanoma metastases}

For their therapeutic potential to be realised, antimelanoma nanoparticles need to target not only the tumour cells but also reach the sites of metastases. In melanoma, metastases present in a range of tissue types, and every tissue has its own unique challenges for nanoparticle penetration. For many solid tumours, nanoparticle delivery through intravenous administration is promoted by the enhanced permeability and retention effect. ${ }^{56}$ Nano-sized carriers escape renal clearance and cannot penetrate the tight junctions of endothelial cells in normal vessels, but can extravasate into tumour tissue with abnormal vascular architecture. High expression of angiogenic factors in tumours such as VEGF leads to hypervascularisation and disorganised vascular architecture. Insufficient lymphatic drainage often leads to accumulation of nanosized objects in tumours. Thus, the enhanced permeability and retention effect can be exploited as a passive targeting mechanism, which has previously been shown for nanoparticles in a murine melanoma model. ${ }^{57}$ The enhanced permeability and retention effect can be increased by the addition of low doses of tumour necrosis factor $\alpha(\mathrm{TNF} \alpha)$, which works as a vasoactive drug that promotes extravasation of the nanoparticles into the tumour. The addition of TNF $\alpha$ plus stealth liposomes has been shown to not only increase their localisation in the tumour by five-to-sixfold in a murine melanoma model, but also to improve their distribution, resulting in better efficacy. ${ }^{58}$

In a model of melanoma metastases to the lung, continued growth of metastatic tumours has been shown to be preferentially located in specific tissue environments close to blood vessels,$^{59}$ suggesting that passive targeting through enhanced permeability and retention could still have some capacity to deliver nanoparticles to metastases in the lung. In addition to intravenous administration, the lungs can also be accessed by aerosolised particles through inhalation. However, loss of enhanced permeability and retention and penetration problems by the nanoparticles through the lung tissue are problems that would need to be addressed before inhalation could be suggested as a more effective administration route than intravenous dosing.

Many fenestrae of the liver vasculature allow the enhanced permeability and retention effect to be exploited for targeting of nanoparticles to liver metastases. However, non-targeted accumulation of nanoparticles in the liver can cause problems for nanocarrier design because hepatotoxicity is a common side-effect of many chemotherapeutic drugs, including dacarbazine. ${ }^{60}$ For this reason, receptor-mediated uptake of tumour cell-targeting nanoparticles is the best approach to reduce drug dispersal in surrounding hepatic tissue and limit toxic side-effects. Indeed, anticancer drug-loaded nanoparticles have been developed that target CD44 receptors overexpressed in tumour tissue in a liver implant model of colon cancer ${ }^{6}$ and since the same targeting mechanism has been used to successfully deliver nanoparticles to melanoma cells in vivo, ${ }^{62}$ this mechanism could be used for the delivery of drug nanocarriers to hepatic melanoma metastases.

The blood-brain barrier, which allows for highly selected molecule exchange, poses distinct challenges for intravenous administration of nanoparticles when targeting the brain. Fortunately, the architecture of the tumour vasculature can disrupt this barrier function, which means that the enhanced permeability and retention effect can still be exploited. Indeed, ultrasmall superparamagnetic iron oxide nanoparticles can enhance detection of intracerebral melanoma lesions in mice, compared with other contrast agents, ${ }^{63}$ where the enhanced permeability and retention effect is the likely mechanism of superparamagnetic iron oxide nanoparticle accumulation in the brain.

Growth of metastases in the regional lymph nodes and the afferent lymphatic vessels (resulting in satellite or in-transit metastases in the skin), can lead to substantial morbidity. Therefore, targeting of disseminated melanoma cells in the lymphovascular system by nanoparticles also has huge therapeutic potential. Recently, a phase 2 clinical study of patients with stage III-IV melanoma showed that nano-based immunotherapy combined with different adjuvants given subcutaneously, topically, or by intra-lymph node injection promoted combined memory and effector CD8+ T-cell responses in patients..$^{64,65}$ Overall, targeting to the lymphatic system through intravenous administration is often a passive process because nanoparticles can be transported there by leucocytes as part of a normal functioning immune response. ${ }^{66}$ However, accumulation can be improved further by promotion of leucocyte uptake, mainly through surface modifications of the nanoparticles, including carbohydrate coating, ${ }^{67}$ immunoglobulin conjugation, ${ }^{68}$ and negative surface charge. ${ }^{66}$ 


\section{Considerations for the development of highly eff ective anti-melanoma nanoparticles for clinical use}

Nano-based therapeutics off er distinct advantages over conventional drug treatments in that they can provide multifunctional combinations of targeting ability, diagnostic value, and therapeutic capacity (ie, thermoablation, acoustic ablation, and multiple drug delivery) in one unique particle. For the development of successful treatments, individual cancer specifi cities should be addressed, including eff ective penetration to the sites of metastasis, identifi cation of suitable cell surface targeting moieties, eff ects of the tumour microenvironment composition, and drug dosing requirements for eff ective tumour ablation.

Although clinical translation of nanoformulations based on inorganic nanoparticles such as iron oxide as diagnostic devices in oncology is quite advanced, only a few examples exist of targeted nanotherapies for metastatic cancer of theranostic nanoparticles in clinical trials (eg, the CALAA-01 trial $^{13,44}$ ). In April, 2013, a very promising phase 2 study (NCT01812746) for establishing the effi cacy of targeted polymeric nanoparticles containing docetaxel (BIND-014) in patients with metastatic castration-resistant prostate cancer started in the USA. ${ }^{69}$ The paucity of examples of sophisticated theranostic nanoparticles in the clinic is due to many reasons that are not only related to efficacy, but also poor stability and high manufacturing costs for the scale-up of such complex nanoformulations (panel), a rationale for which has been reviewed recently. ${ }^{70}$ These issues need to be addressed in the design of successful theranostic nanoparticles for the treatment of late-stage melanoma.

In the treatment of gross metastasis, eff ective tumourspecifi c targeting in melanoma patients is essential for the development of nanotherapies that are sufficiently eff ective to cure the disease; both targeting of the nanoparticles to the metastatic site and to the tumour cells directly. The passive eff ect of enhanced permeability and retention-mediated nanoparticle accumulation in tumours is now recognised to present too many inconsistencies to be relied on for eff ective targeting to metastatic melanoma tumours. ${ }^{71}$ Although the enhanced permeability and retention eff ect can be augmented by angiotensin II-induced vasoconstriction in cancer patients, ${ }^{72}$ prediction of the level of nanoparticle accumulation at the site of metastases, promoted by the enhanced permeability and retention eff ect alone, is diffi cult. It is also ineffective for nanoparticle accumulation if metastatic tumours are small and poorly vascularised, especially in micro-metastases (tumours <2 $\mathrm{mm}$ in size). Furthermore, interstitial fi brosis, which often occurs with melanoma metastases, can limit the diff usion of compounds and drug carriers to tumours from the vasculature. Therefore, improved targeting to secondary tumour sites is necessary to develop nanoparticle therapies with increased effi cacy for patients with melanoma. Recently, Ruoslahti and colleagues $^{73}$ identified peptides that recognise specific markers associated with tumour vessels. They engineered di-block peptides, which first recognise the tumour vasculature and are then cleaved intracellularly to expose new sequences targeting specific cell pathways that enhance tumour penetration. This strategy has been shown to promote tumour penetration in different tumour murine models of both conventional anticancer drugs and nanoparticles co-added with such peptides. ${ }^{73}$ Similar mechanisms to enhance extravasation in poorly vascularised tumours together with direct targeting to secondary tumour sites are needed to develop nanoparticle therapies with increased efficacy for patients with melanoma.

In view of the diverse metastatic spread of melanoma cells to a wide range of organs, the ideal goal is to selectively target melanoma tumours and provide potent cytotoxic drug delivery, without affecting other tissue types-a so-called homing toxic bomb. Such nanoparticles would provide improved dosing and greatly reduced systemic toxicity, with more control over the drug load that can be given to and tolerated by the patient. To achieve this aim, homing toxic bombs must be decorated with targeting molecules that provide recognition of suitable cell surface markers on melanoma cells (appendix p 2). However, selection of one targeting moiety will probably be insufficient for long-term effective treatment of late-stage melanoma, because of the high propensity for tumour cells to acquire resistance mechanisms. Thus, the identification of several melanoma cell surface markers that are suitable for nanoparticle targeting will be essential to provide long-

\section{Panel: Advantages and disadvantages of the use of nanotherapeutics for} the treatment of metastatic melanoma over conventional drug therapies

\section{Advantages}

- Anti-melanoma drug-carrying capacity of one or more compound. Hydrophillic and hydrophobic drugs can be incorporated simultaneously.

- Improved systemic circulation of drugs and enhanced tissue permeability to improve the bioavailability of anti-melanoma chemotherapeutics to metastatic tumours.

- Simultaneous imaging and therapeutic properties for the diagnosis and treatment of metastatic disease.

- Controlled release of anti-melanoma drug(s) inside the tumour cells.

- Successful targeting to reduce systemic toxicity of chemotherapeutic agents, thereby reducing unwanted side-effects.

- Melanoma patients can be protected from developing multidrug resistance by reducing the drug efflux capacity of cancer cells.

Disadvantages

- Higher manufacturing costs since scale-up can be encumbered because of complex nanomaterial formulation.

- Unwanted activation of immune response leading to allergic reactions. Toxicity levels of new formulations must be carefully assessed before patient trials.

- Removal of nanoparticles by the reticuloendothelial system and other clearance mechanisms. Nanoparticles must be engineered to reduce opsonisation and optimise the surface properties to reduce clearance. 

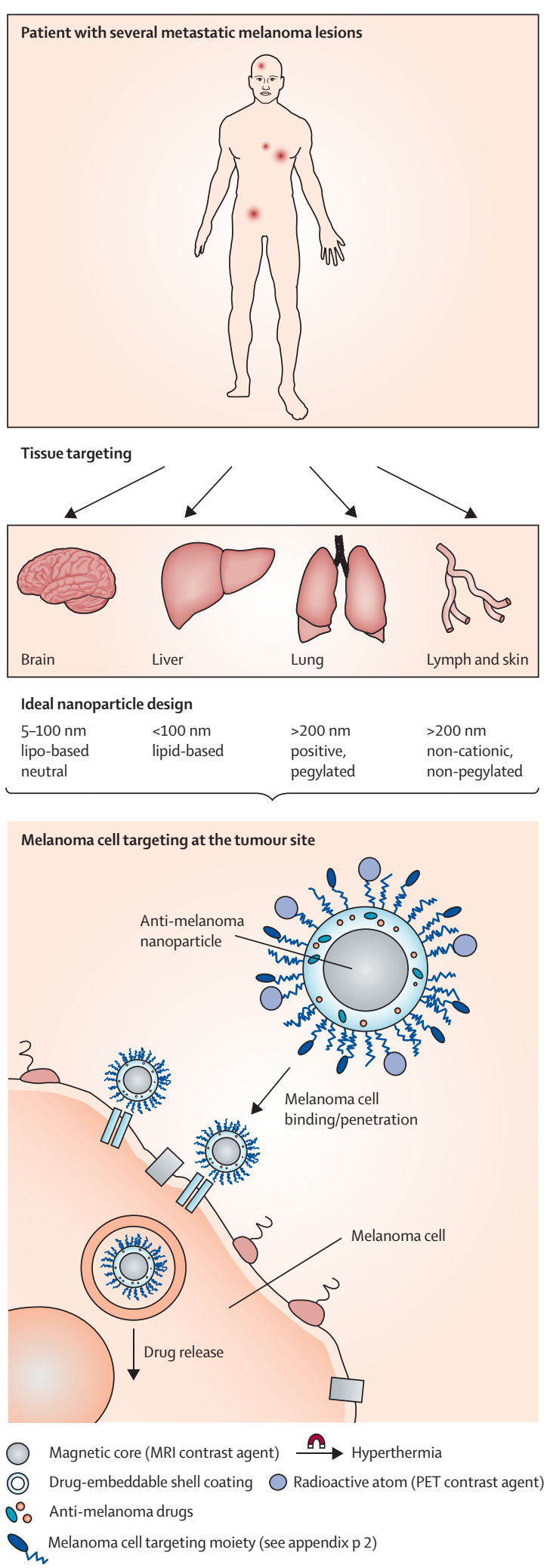

Figure 4: Schematic strategy of effective nanotherapies for advanced stage melanoma term disease-free survival. Therefore, future efforts should focus on the identification of such markers.

The technology now exists to synthesise nanoparticles with wide-ranging geometries (figure 3), to offer more options to enhance tissue targeting. For example, different sized nanoparticles will preferentially target individual tissue types. Nanoparticles less than $100 \mathrm{~nm}$ in size will favour brain and liver targeting, whereas systemic intravenous administration of nanoparticles larger than $200 \mathrm{~nm}$ will be trapped in pulmonary capillaries, thereby favouring lung targeting. ${ }^{74}$ However, nanoparticles larger than $200 \mathrm{~nm}$ are generally sequestered by the reticuloendothelial system, enhancing accumulation in the liver and spleen, with obvious implications for toxicity. Thus, these nanoparticles might not be an optimum choice unless specific properties of the engineered nanoparticles allow them to escape the reticuloendothelial system (ie, reduced interaction with the opsonins).$^{75}$ In this regard, surface functionalisation also has a huge effect on nanoparticle accumulation. For example, non-pegylated varieties will be recognised by leucocytes and transported to the lymph, where avoidance of this trafficking is essential for targeting to other organs. $^{76}$ Other modifications involving shape, net charge, and surface lipids will also affect tissue targeting, and will favour accumulation in some organs over others. However, some tissues, such as bone, have been researched poorly so far in terms of nanoparticle targeting and need more attention to achieve effective targeting to melanoma metastases at these sites. Manipulation of these considerations for nanoparticle delivery, coupled with selective targeting to melanoma cells, will undoubtedly enhance the capacity of delivery to specific organs. Thus, how nanoparticles are used to treat metastatic melanoma could necessitate tailoring of formulations on the basis of individual patients' needs.

Overall diversity in the requirements of nanoparticle designs for specific organ targeting is challenging for the development of nanotherapeutics to treat melanoma, since this cancer can disseminate to several organs during metastasis. Successful targeting to all these metastatic locations is, of course, desired, but would ultimately need the production of mixed batches of nanoparticles with a range of properties that can simultaneously target many tissues. However, this need poses engineering problems, where at the sub- $\mu \mathrm{m}$ scale distinct shapes can dramatically change interfacial interactions, ${ }^{\pi}$ which makes it difficult to predict how heterogeneous populations of nanoparticles would behave in patients. Therefore, the existing consensus in nanomedicine is to develop homogeneous, monodispersed nanoparticle preparations for drug delivery. ${ }^{78}$ However, such preparations are unlikely to be sufficient to target melanoma metastases in several anatomical locations. Therefore, we propose that nanotherapeutic treatment strategies for late stage melanoma could require several doses of nanoparticles, with different 


\section{Search strategy and selection criteria}

References were obtained from searches of PubMed and Web of Knowledge with the following medical subject heading search terms: "melanoma", "anti-CTLA4 antibody", "BRAF inhibitors", "nanotechnology", "nanomedicine", "nanoparticles", "theranostic", "nanotherapeutics", "contrast agents", and "EPR effect". These searches were required for specific areas of discussion in the Review, and only articles published since 1980, in English, have been included. Clinical trial information was found on the ClinicalTrials.gov website (www.clinicaltrials. gov) with the search criteria "melanoma and nanoparticles".

properties in each dose to eff ectively target all metastatic tumours in patients. Although this idea is analogous to the combinatorial therapy approach, nanoparticles have the distinct advantage of providing specific organ targeting and reducing eff ective drug clearance. The panel provides a summary of these advantages and disadvantages for the use of nanotherapies in the treatment of melanoma. Overall, the advantages clearly outweigh the disadvantages, suggesting that nanomaterials have huge potential for the successful treatment of metastatic melanoma if researchers successfully address the considerations discussed here.

Figure 4 summarises these considerations to produce future nanoformulations for the successful treatment of metastatic melanoma. Future research efforts should focus on such considerations so that the promise of nanotherapies for metastatic melanoma treatment can be realised.

\section{Summary}

Although some breakthroughs have recently been achieved in the treatment of metastatic melanoma, the disease still remains largely incurable. Alarmingly, melanoma incidence rates are rising faster than those of any other tumour type and researchers need to develop therapies to eff ectively deal with the disease after metastatic dissemination. Nanotechnology has the potential capability to achieve this goal. Indeed, nanomedicine promises to revolutionise the way that metastatic tumours are treated in the future. Only a few trials have investigated nanomedicine for melanoma so far, but in view of the amount of preclinical work that is underway, we predict that the number of clinical studies is set to rise dramatically in the next few years. For these new therapies to be successful, their development requires a multidisciplinary approach at an early stage in their design that incorporates strong interactions between scientists (including cell biologists, chemists, pharmacologists, toxicologists, engineers, and immunologists) and clinicians (including surgeons, oncologists, and histopathologists), to develop highly effective antimelanoma nanoparticles to improve patient outcome. Overall, the potential of nanotherapeutics offers huge scope for dramatically increasing overall survival for melanoma patients, as long as a multidisciplinary approach is taken to develop this technology for the clinic.

\section{Contributors}

FBB, MM, and VS wrote and designed the report. FBB and VS designed and prepared the figures and panel. CAW and VS prepared the table and appendix.

\section{Conflicts of interest}

We declare that we have no conflicts of interest.

\section{Acknowledgments}

This work was supported by funds from The British Skin Foundation and The Royal Society. We thank Grant Wheeler for his helpful comments during the preparation of this article.

\section{References}

1 Lens MB, Dawes M. Global perspectives of contemporary epidemiological trends of cutaneous malignant melanoma. Br J Dermatol 2004; 150: 179-85.

2 Rigel DS, Russak J, Friedman R. The evolution of melanoma diagnosis: 25 years beyond the ABCDs. CA Cancer J Clin 2010; 60: 301-16.

3 Balch CM, Gershenwald JE, Soong SJ, et al. Final version of 2009 AJCC melanoma staging and classification. J Clin Oncol 2009; 27: 6199-206.

4 Hodi FS, O’Day SJ, McDermott DF, et al. Improved survival with ipilimumab in patients with metastatic melanoma. N Engl J Med 2010; 363: 711-23.

5 Chapman PB, Hauschild A, Robert C, et al. Improved survival with vemurafenib in melanoma with BRAF V600E mutation. N Engl J Med 2011; 364: 2507-16.

6 Robert C, Thomas L, Bondarenko I, et al. Ipilimumab plus dacarbazine for previously untreated metastatic melanoma. N Engl J Med 2011; 364: 2517-26.

7 Mura S, Couvreur P. Nanotheranostics for personalized medicine. Adv Drug Deliv Rev 2012; 64: 1394-416.

8 Finn L, Markovic SN, Joseph RW. Therapy for metastatic melanoma: the past, present, and future. BMC Med 2012; 10: 23

9 Shada AL, Molhoek KR, Slingluff CL Jr. Interface of signal transduction inhibition and immunotherapy in melanoma. Cancer J 2010; 16: 360-66.

10 Nikolaou VA, Stratigos AJ, Flaherty KT, Tsao H. Melanoma: new insights and new therapies. J Invest Dermatol 2012; 132 (3 pt 2): 854-63.

11 Gottesman MM. Mechanisms of cancer drug resistance. Annu Rev Med 2002; 53: 615-27.

12 Di Maio M, Chiodini P, Georgoulias V, et al. Meta-analysis of single-agent chemotherapy compared with combination chemotherapy as second-line treatment of advanced non-small-cell lung cancer. J Clin Oncol 2009; 27: 1836-43.

13 Deng WG, Kwon J, Ekmekcioglu S, Poindexter NJ, Grimm EA. IL-24 gene transfer sensitizes melanoma cells to erlotinib through modulation of the Apaf-1 and Akt signaling pathways. Melanoma Res 2010; 21: 44-56.

14 Schicher N, Paulitschke V, Swoboda A, et al. Erlotinib and bevacizumab have synergistic activity against melanoma. Clin Cancer Res 2009; 15: 3495-502.

15 White RM, Cech J, Ratanasirintrawoot S, et al. DHODH modulates transcriptional elongation in the neural crest and melanoma. Nature 2011; 471: 518-22.

16 Stecca B, Mas C, Clement V, et al. Melanomas require HEDGEHOG-GLI signaling regulated by interactions between GLI1 and the RAS-MEK/AKT pathways. Proc Natl Acad Sci USA 2007; 104: 5895-900

17 Sharma P, Singh A, Brown SC, et al. Multimodal nanoparticulate bioimaging contrast agents. Methods Mol Biol 2010; 624: 67-81.

18 Nappini S, Bombelli FB, Bonini M, Norden B, Baglioni P. Magnetoliposomes for controlled drug release in the presence of low-frequency magnetic field. Soft Matter 2010; 6: 154-62.

19 Choi KY, Liu G, Lee S, Chen XY. Theranostic nanoplatforms for simultaneous cancer imaging and therapy: current approaches and future perspectives. Nanoscale 2012; 4: 330-42. 
20 Shapira A, Livney YD, Broxterman HJ, Assaraf YG. Nanomedicine for targeted cancer therapy: towards the overcoming of drug resistance. Drug Resist Update 2011; 14: 150-63.

$21 \mathrm{Hu}$ CMJ, Zhang LF. Nanoparticle-based combination therapy toward overcoming drug resistance in cancer. Biochem Pharmacol 2012; 83: 1104-11.

22 Arora HC, Jensen MP, Yuan Y, et al. Nanocarriers enhance doxorubicin uptake in drug-resistant ovarian cancer cells Cancer Res 2012; 72: 769-78.

23 Ding B, Wu X, Fan W, et al. Anti-DR5 monoclonal antibodymediated DTIC-loaded nanoparticles combining chemotherapy and immunotherapy for malignant melanoma: target formulation development and in vitro anticancer activity. Int J Nanomedicine 2011; 6: 1991-2005

24 Shelley WB, Talanin N, Shelley ED. Polysorbate 80 hypersensitivity. Lancet 1995; 345: 1312-13.

25 Miele E, Spinelli GP, Tomao F, Tomao S. Albumin-bound formulation of paclitaxel (Abraxane ABI-007) in the treatment of breast cancer. Int J Nanomedicine 2009; 4: 99-105.

26 Kottschade LA, Suman VJ, Amatruda T, et al. A phase II trial of nab-paclitaxel (ABI-007) and carboplatin in patients with unresectable stage IV melanoma: a North Central Cancer Treatment Group Study, N057E(1). Cancer 2011; 117: 1704-10.

27 Ernsting MJ, Tang WL, MacCallum N, Li SD. Synthetic modification of carboxymethylcellulose and use thereof to prepare a nanoparticle forming conjugate of docetaxel for enhanced cytotoxicity against cancer cells. Bioconjug Chem 2011; 22: 2474-86.

28 Ernsting MJ, Murakami M, Undzys E, Aman A, Press B, Li SD A docetaxel-carboxymethylcellulose nanoparticle outperforms the approved taxane nanoformulation, Abraxane, in mouse tumor models with significant control of metastases. J Control Release 2012; 162: 575-81.

29 Chaudhuri P, Paraskar A, Soni S, Mashelkar RA, Sengupta S. Fullerenol-cytotoxic conjugates for cancer chemotherapy. ACS Nano 2009; 3: 2505-14

30 Zheng D, Li X, Xu H, Lu X, Hu Y, Fan W. Study on docetaxelloaded nanoparticles with high antitumor efficacy against malignant melanoma. Acta Biochim Biophys Sin (Shanghai) 2009, 41: 578-87.

31 Parhi P, Mohanty C, Sahoo SK. Nanotechnology-based combinational drug delivery: an emerging approach for cancer therapy. Drug Discov Today 2012; 17: 1044-52.

32 Dilnawaz F, Singh A, Mohanty C, Sahoo SK. Dual drug loaded superparamagnetic iron oxide nanoparticles for targeted cancer therapy. Biomaterials 2010; 31: 3694-706.

33 Liu XQ, Song WJ, Sun TM, Zhang PZ, Wang J. Targeted delivery of antisense inhibitor of miRNA for antiangiogenesis therapy using cRGD-functionalized nanoparticles. Mol Pharm 2011; 8: 250-59.

34 Torchilin VP. Tat peptide-mediated intracellular delivery of pharmaceutical nanocarriers. Adv Drug Deliv Rev 2008; 60: 548-58.

35 Lentacker I, Geers B, Demeester J, De Smedt SC, Sanders NN. Design and evaluation of doxorubicin-containing microbubbles for ultrasound-triggered doxorubicin delivery: cytotoxicity and mechanisms involved. Mol Ther 2010; 18: 101-08.

36 Lentacker I, Wang N, Vandenbroucke RE, Demeester J, De Smedt SC, Sanders NN. Ultrasound exposure of lipoplex loaded microbubbles facilitates direct cytoplasmic entry of the lipoplexes. Mol Pharm 2009; 6: 457-67.

37 Soman N, Marsh J, Lanza G, Wickline S. New mechanisms for non-porative ultrasound stimulation of cargo delivery to cell cytosol with targeted perfluorocarbon nanoparticles. Nanotechnology 2008; 19: 185102.

38 Harada Y, Ogawa K, Irie Y, et al. Ultrasound activation of TiO2 in melanoma tumors. J Control Release 2011; 149: 190-95.

39 Sawant RR, Sriraman SK, Navarro G, Biswas S, Dalvi RA Torchilin VP. Polyethyleneimine-lipid conjugate-based $\mathrm{pH}$-sensitive micellar carrier for gene delivery. Biomaterials 2012; 33: 3942-51.

40 Perche F, Benvegnu T, Berchel M, et al. Enhancement of dendritic cells transfection in vivo and of vaccination against B16F10 melanoma with mannosylated histidylated lipopolyplexes loaded with tumor antigen messenger RNA. Nanomedicine 2011; 7: 445-53.

41 Yang T, Wang Y, Li Z, et al. Targeted delivery of a combination therapy consisting of combretastatin A4 and low-dose doxorubicin against tumor neovasculature. Nanomedicine 2012; 8: 81-92.
42 Chen Y, Bathula SR, Yang Q, Huang L. Targeted nanoparticles deliver siRNA to melanoma. J Invest Dermatol 2010; 130: 2790-98.

43 Davis ME, Zuckerman JE, Choi CH, et al. Evidence of RNAi in humans from systemically administered siRNA via targeted nanoparticles. Nature 2010; 464: 1067-70.

44 Bartlett DW, Davis ME. Impact of tumor-specific targeting and dosing schedule on tumor growth inhibition after intravenous administration of siRNA-containing nanoparticles. Biotechnol Bioeng 2008; 99: 975-85.

45 Hildebrandt B, Wust P, Ahlers O, et al. The cellular and molecular basis of hyperthermia. Crit Rev Oncol Hemat 2002; 43: 33-56.

$46 \mathrm{Lu} \mathrm{W}$, Xiong C, Zhang G, et al. Targeted photothermal ablation of murine melanomas with melanocyte-stimulating hormone analog-conjugated hollow gold nanospheres. Clin Cancer Res 2009; 15: 876-86.

47 Balivada S, Rachakatla RS, Wang HW, et al. A/C magnetic hyperthermia of melanoma mediated by iron(0)/iron oxide core/shell magnetic nanoparticles: a mouse study. BMC Cancer 2010; 10: 119.

48 Rachakatla RS, Balivada S, Seo GM, et al. Attenuation of mouse melanoma by a/c magnetic field after delivery of bi-magnetic nanoparticles by neural progenitor cells. ACS Nano 2010; 4: 7093-104.

49 Ishii-Osai Y, Yamashita T, Tamura Y, et al. N-propionyl-4-Scysteaminylphenol induces apoptosis in B16F1 cells and mediates tumor-specific T-cell immune responses in a mouse melanoma model. J Dermatol Sci 2012; 67: 51-60.

50 Kim C, Cho EC, Chen J, et al. In vivo molecular photoacoustic tomography of melanomas targeted by bioconjugated gold nanocages. ACS Nano 2010; 4: 4559-64.

51 Benezra M, Penate-Medina O, Zanzonico PB, et al. Multimodal silica nanoparticles are effective cancer-targeted probes in a model of human melanoma. J Clin Invest 2011; 121: 2768-80.

52 Faries MB, Thompson JF, Cochran A, et al. The impact on morbidity and length of stay of early versus delayed complete lymphadenectomy in melanoma: results of the Multicenter Selective Lymphadenectomy Trial (I). Ann Surg Oncol 2010; 17: 3324-29.

53 Lim SW, Kim HW, Jun HY, et al. TCL-SPION-enhanced MRI for the detection of lymph node metastasis in murine experimental model. Acad Radiol 2011; 18: 504-11.

54 Bradbury MS, Phillips E, Montero PH, et al. Clinically-translated silica nanoparticles as dual-modality cancer-targeted probes for image-guided surgery and interventions. Integr Biol 2012; 5: 74-86.

55 Vannucci L, Falvo E, Fornara M, et al. Selective targeting of melanoma by PEG-masked protein-based multifunctional nanoparticles. Int J Nanomedicine 2012; 7: 1489-509.

56 Maeda H. Macromolecular therapeutics in cancer treatment: the EPR effect and beyond. J Control Release 2012; 164: 138-44.

57 Okuda T, Kawakami S, Higuchi Y, et al. Enhanced in vivo antitumor efficacy of fenretinide encapsulated in polymeric micelles. Int J Pharm 2009; 373: 100-06.

58 Seynhaeve AL, Hoving S, Schipper D, et al. Tumor necrosis factor alpha mediates homogeneous distribution of liposomes in murine melanoma that contributes to a better tumor response. Cancer Res 2007; 67: 9455-62.

59 Cameron MD, Schmidt EE, Kerkvliet N, et al. Temporal progression of metastasis in lung: cell survival, dormancy, and location dependence of metastatic inefficiency. Cancer Res 2000; 60: 2541-46.

60 Paschke R, Heine M, Braun S, Usadel KH. Mechanisms of hepatotoxicity caused by dacarbazine in rats. J Cancer Res Clin Oncol 1993; 119: 475-81.

61 Choi KY, Jeon EJ, Yoon HY, et al. Theranostic nanoparticles based on PEGylated hyaluronic acid for the diagnosis, therapy and monitoring of colon cancer. Biomaterials 2012; 33: 6186-93.

62 Jin YJ, Termsarasab U, Ko SH, et al. Hyaluronic acid derivativebased self-assembled nanoparticles for the treatment of melanoma. Pharm Res 2012; 29: 3443-54.

63 Leenders W, Kusters B, Pikkemaat J, et al. Vascular endothelial growth factor-A determines detectability of experimental melanoma brain metastasis in GD-DTPA-enhanced MRI. Int J Cancer 2003; 105: 437-43.

64 Speiser D, Schwarz K, Baumgaertner P, et al. Memory and effector CD8 T-cell responses after nanoparticle vaccination of melanoma patients. J Immunother 2010; 33: 848-58. 
65 Goldinger S, Dummer R, Baumgaertner P, et al. Nano-particle vaccination combined with TLR-7 and -9 ligands triggers memory and effector Cd8+ T-cell responses in melanoma patients. Eur J Immunol 2012; 42: 3049-61.

66 Raz A, Bucana C, Fogler WE, Poste G, Fidler IJ. Biochemical, morphological, and ultrastructural studies on the uptake of liposomes by murine macrophages. Cancer Res 1981; 41: 487-94.

67 Tassa C, Shaw SY, Weissleder R. Dextran-coated iron oxide nanoparticles: a versatile platform for targeted molecular imaging, molecular diagnostics, and therapy. Acc Chem Res 2011; 44: 842-52.

68 Finkelstein MC, Kuhn SH, Schieren H, Weissmann G, Hoffstein S. Liposome uptake by human leukocytes. Enhancement of entry mediated by human serum and aggregated immunoglobulins. Biochim Biophys Acta 1981; 673: 286-302.

69 Hrkach J, Von Hoff D, Ali MM, et al. Preclinical development and clinical translation of a PSMA-targeted docetaxel nanoparticle with a differentiated pharmacological profile. Sci Transl Med 2012; 4: 128 ra39.

70 Desai N. Challenges in development of nanoparticle-based therapeutics. AAPS J 2012; 14: 282-95.

71 Prabhakar U, Blakey DC, Maeda H, et al. Challenges and key considerations of the enhanced permeability and retention effect (EPR) for nanomedicine drug delivery in oncology. Cancer Res 2013; 73: 2412-17.
72 Maeda H. Vascular permeability in cancer and infection as related to macromolecular drug delivery, with emphasis on the EPR effect for tumor-selective drug targeting. Proc Jpn Acad Ser B Phys Biol Sci 2012; 88: 53-71.

73 Alberici L, Roth L, Sugahara KN, et al. De novo design of a tumor-penetrating peptide. Cancer Res 2013; 73: 804-12.

74 Schroeder A, Heller DA, Winslow MM, et al. Treating metastatic cancer with nanotechnology. Nat Rev Cancer 2012; 12: 39-50.

75 Karmali PP, Simberg D. Interactions of nanoparticles with plasma proteins: implication on clearance and toxicity of drug delivery systems. Expert Opin Drug Deliv 2011; 8: 343-57.

76 Alexis F, Pridgen E, Molnar LK, Farokhzad OC. Factors affecting the clearance and biodistribution of polymeric nanoparticles. Mol Pharm 2008; 5: 505-15.

77 Gissi R, Decuzzi P. The effect of shape and size in micro-/ nanodimples adhesion. J Appl Physiol 2005; 98: 014310.

78 Petros RA, Desimone JM. Strategies in the design of nanoparticles for therapeutic applications. Nat Rev Drug Discov 2010; 9: 615-27. 